\title{
Strong temperature dependence of water reorientation in hydrophobic hydration shells
}

\author{
C. Petersen, ${ }^{a}{ }^{2}$ K.-J. Tielrooij, and H. J. Bakker \\ FOM Institute for Atomic and Molecular Physics, Science Park 113, 1098 XG Amsterdam, The Netherlands
}

(Received 23 January 2009; accepted 28 April 2009; published online 5 June 2009)

\begin{abstract}
We study the temperature dependence of the orientational mobility of water molecules solvating hydrophobic molecular groups with femtosecond midinfrared spectroscopy. We observe that these dynamics show a strong temperature dependence. At temperatures $<30^{\circ} \mathrm{C}$ the solvating water molecules show a reorientation time $>10 \mathrm{ps}$, which is more than four times slower than in bulk water. With increasing temperature, the reorientation of the solvating molecules strongly accelerates and becomes much more equal to the reorientation rate of the molecules in the bulk liquid. These observations indicate that water molecules form relatively rigid solvation structures around hydrophobic molecular groups that melt at elevated temperatures. (C) 2009 American Institute of Physics. [DOI: 10.1063/1.3142861]
\end{abstract}

\section{INTRODUCTION}

The spatial structure of large (bio)molecular structures in aqueous solution is strongly determined by the so-called hydrophobic effect, i.e., the tendency of a polar groups to associate in aqueous solution. This association interaction is strongly connected to the mutual interactions of water molecules near hydrophobic molecular groups. In the 1940s Frank and Evans ${ }^{1}$ studied the thermodynamics of dissolving hydrophobic molecules in water. Naively one would expect that the introduction of a weakly interacting hydrophobic solute in water leads to a break-up of strong water-water hydrogen bonds and an increase in the disorder of the water network. Hence, the dissolution of a hydrophobic solute is expected to lead to an increase of both the enthalpy $\Delta H$ and the excess entropy $\Delta S_{\text {exc }}$ (the entropy contribution other than the translational entropy). However, Frank and Evans observed that the dissolution of hydrophobic compounds is associated with negative changes in both the enthalpy and the excess entropy $\left(\Delta H<0, \Delta S_{\text {exc }}<0\right)$. Both effects indicate that the introduction of hydrophobic groups leads to an increased structuring of the surrounding water molecules. These structures were denoted as hydrophobic icebergs.

In contrast to the thermodynamic findings, molecular scale studies did not find the structure of water surrounding hydrophobic groups to be very different from bulk liquid water. For instance, in neutron scattering studies the oxygenoxygen distances of the solvating water molecules were observed to be similar as in bulk liquid water. ${ }^{2-5}$ However, the dynamics of water molecules surrounding hydrophobic groups were observed to be very different from bulk liquid water. In both NMR and femtosecond midinfrared spectroscopy measurements, it was observed that the solvating water molecules show much slower orientational dynamics than the molecules in bulk liquid water. ${ }^{6-13}$

The thermodynamic properties of hydrophobic hydration

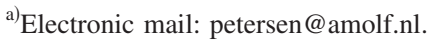

are strongly temperature dependent. ${ }^{14-19}$ For small hydrophobic solutes, the free energy change associated with the solvation reaches a maximum at an elevated temperature, which implies that at this temperature the change in excess entropy has become zero, and the enthalpy change has become positive. ${ }^{15,17-19}$ The increase in $\Delta H$ and $\Delta S_{\text {exc }}$ with temperature suggests that the water structure surrounding the hydrophobic molecule becomes disrupted, i.e., a "melting" of the hydrophobic hydration shells. Here we study the temperature dependence of the dynamics of the hydrophobic hydration shells using polarization-resolved femtosecond midinfrared spectroscopy. ${ }^{20-30}$ We observe that heating of the solution leads to a strong acceleration of the molecular reorientation of the hydrophobic hydration structures, which indicates that these structures become much more labile.

\section{EXPERIMENTAL METHODS}

We studied the orientational dynamics of water molecules in aqueous solutions of tetramethylurea (TMU) and tertiary butyl alcohol (TBA) at different temperatures. We added $4 \%$ heavy water $\left(\mathrm{D}_{2} \mathrm{O}\right)$ to the water, which leads to a solution of $8 \%$ HDO in $\mathrm{H}_{2} \mathrm{O}$. There will also be a small amount of $\mathrm{D}_{2} \mathrm{O}$ in the sample $(\sim 0.02 \%)$. The signal from HDO will be a factor of $\sim 40$ larger than that arising from $\left(\mathrm{D}_{2} \mathrm{O}\right)$ and we can disregard this contribution in the analysis. The O-D vibration with a strong absorption around $4 \mu \mathrm{m}$ $\left(2500 \mathrm{~cm}^{-1}\right)$ of the HDO molecules is used as a local probe for the orientational dynamics of the water hydroxyl groups. The orientational dynamics of the O-D groups is measured using polarization-resolved pump-probe spectroscopy.

The pulses required for the polarization-resolved pumpprobe experiment are generated via several frequency conversion processes. ${ }^{12,26,27}$ We use $50 \%$ of a commercial Ti:sapphire laser system $(800 \mathrm{~nm}, 100 \mathrm{fs}, 1 \mathrm{~mJ}, 1 \mathrm{kHz})$ to pump a white-light seeded optical parametric amplification (OPA) in $\beta$-barium borate (BBO). The OPA is tuned to produce idler pulses with a wavelength of $2.0 \mu \mathrm{m}$. These pulses are frequency-doubled in a second BBO crystal. Subse- 
quently, the pulses are difference-frequency mixed in a $\mathrm{KNbO}_{3}$ crystal, using the remaining $50 \%$ of the $800 \mathrm{~nm}$ light. This process yields midinfrared pulses that are resonant with the O-D stretch vibration of HDO, having a duration of $150 \mathrm{fs}$, a wavelength of $4 \mu \mathrm{m}$ and an energy of a few microjoules.

The midinfrared light is coupled into a pump-probe setup. A small fraction of the light is split off by a wedged $\mathrm{CaF}_{2}$ window to obtain probe and reference pulses. The transmitted light forms the pump beam, and using a $\lambda / 2$ plate, its polarization is set to $45^{\circ}$ with respect to that of the probe beam. The pump is sufficiently strong to excite a few percent of the O-D stretch vibration to the first excited vibrational state. This excitation results in increased transmission at the frequency of the fundamental $v=0 \rightarrow 1$ transition and induced absorption at the $v=1 \rightarrow 2$ transition. These transmission changes are monitored with the time delayed probe pulse.

The pump, probe, and reference beams are focused onto the sample by an off-axis parabolic mirror and are recollimated by an identical mirror that is placed after the sample. The probe and reference beams are focused onto the entrance slit of a spectrometer, which disperses the beams onto a 2 $\times 32$ liquid-nitrogen-cooled mercury-cadmium-telluride array. Before entering the spectrometer, the probe beam passes through a polarizer allowing the selection of either its parallel or perpendicular polarization component with respect to the pump polarization. This selection results in transient absorptions $\Delta \alpha_{\|}(\omega, t)$ and $\Delta \alpha_{\perp}(\omega, t)$, respectively. These two signals are initially different because of the preferential excitation of HDO molecules that have their OD groups aligned parallel to the pump polarization. As the delay between the pump and probe pulses is increased, molecular reorientation causes the molecules to loose memory of their initial orientation, and the difference between the two signals vanishes. The signals $\Delta \alpha_{\|}(t)$ and $\Delta \alpha_{\perp}(t)$ can be combined to yield the anisotropy,

$$
R(t)=\frac{\Delta \alpha_{\|}(t)-\Delta \alpha_{\perp}(t)}{\Delta \alpha_{\|}(t)+2 \Delta \alpha_{\perp}(t)},
$$

which is a quantity whose decay reflects the reorientation of the observed vibration. The isotropic signal,

$$
\Delta \alpha_{\text {iso }}(t)=\frac{1}{3}\left(\Delta \alpha_{\|}(t)+2 \Delta \alpha_{\perp}(t)\right),
$$

is free of reorientational processes and reflects the decay of the excitation by vibrational relaxation.

\section{RESULTS AND DISCUSSION}

\section{A. Isotropic data}

In Fig. 1, isotropic transient absorption spectra are shown for a solution of $1.5 \mathrm{~m}$ TMU in $\mathrm{HDO}: \mathrm{H}_{2} \mathrm{O}$ at four different delays after exciting the $\mathrm{O}-\mathrm{D}$ stretch vibration with a pump pulse centered at $2500 \mathrm{~cm}^{-1}$. At early delays, the transient spectra show the pump-induced bleaching of the fundamental $v=0 \rightarrow 1$ transition for probe frequencies $>2425 \mathrm{~cm}^{-1}$ and the induced $v=1 \rightarrow 2$ absorption at probe frequencies $<2425 \mathrm{~cm}^{-1}$. At longer delays ( $>3 \mathrm{ps}$ ) transmission changes are observed that persist over the experi-

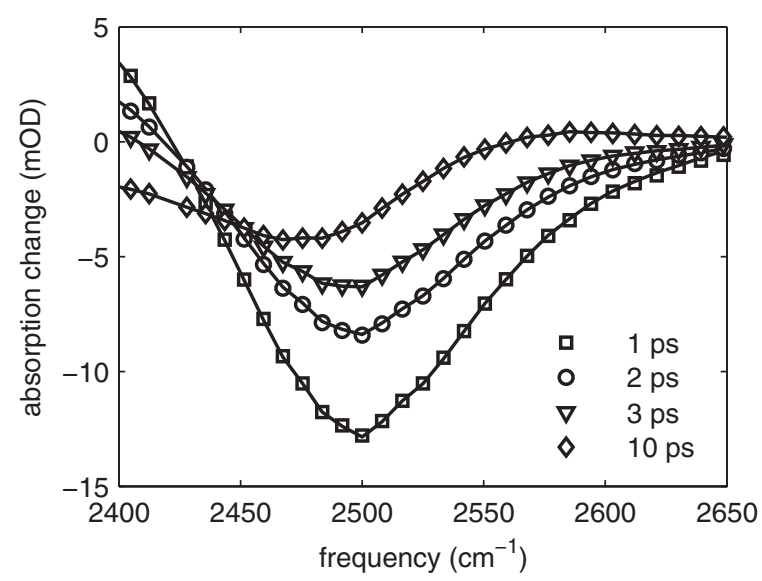

FIG. 1. Transient spectra at four delay times for a solution of $1.5 \mathrm{~mol} / \mathrm{kg}$ TMU in $\mathrm{HDO}: \mathrm{H}_{2} \mathrm{O}$

mentally accessible time range (500 ps) and that have the character of a bleach on the red side of the spectrum and of an induced absorption on the blue side. These persistent transient spectra reflect the temperature rise in the sample that results from the absorption and thermalization of the energy of the pump pulse. A rise in temperature induces a blue shift and a decrease in cross section of the O-D vibration. ${ }^{23,26,27}$

In previous studies of the vibrational relaxation of the $\mathrm{O}-\mathrm{D}$ stretch vibration of $\mathrm{HDO}$ dissolved in $\mathrm{H}_{2} \mathrm{O}$ it was found that the relaxation proceeds via a two-step mechanism in which the energy of the O-D stretch vibration is first transferred with time constant $T_{1}$ to a nonthermal intermediate state followed by a relaxation of this intermediate state with time constant $\tau_{*}$. This second process leads to a complete thermalization of the energy. ${ }^{23,26,27}$ The intermediate state is not observed directly in these experiments, only its effect on the spectrum of the $\mathrm{O}-\mathrm{D}$ stretch is measured.

We model the vibrational relaxation with a model that was developed in Refs. 23, 26, and 31. The two time constants $T_{1}$ and $\tau_{*}$ are treated as global fit parameters, and the cross sections of the transitions between the vibrational levels are allowed to vary over the spectrum. The resulting fits are represented by the solid lines in Figs. 1. The fitted time constants allow us to subtract the time-dependent thermal contribution. In Fig. 2 the isotropic absorption change, where the thermal contribution has been subtracted, is shown as a function of delay for a solution of $1.5 \mathrm{~m}$ TMU at three different temperatures for a probe frequency of $2500 \mathrm{~cm}^{-1}$. The observed bleaching signals show very similar decays, indicating that the vibrational relaxation rate is not strongly temperature dependent.

The resulting time constants $T_{1}$ and $\tau_{*}$ from the fit to the model are presented as a function of temperature in Figs. 3 and 4 . It is seen that $T_{1}$ shows a slight increase with temperature, in agreement with previous findings for the temperature dependence of the vibrational relaxation of the $\mathrm{O}-\mathrm{H}$ stretch vibration of pure $\mathrm{H}_{2} \mathrm{O}$ (Ref. 32) and HDO dissolved in $\mathrm{D}_{2} \mathrm{O}{ }^{33}$ The energy of the excited $\mathrm{O}-\mathrm{D}$ stretch vibration is likely accepted by a combination tone that involves excitation of the HDO bending mode. The increase in $T_{1}$ with temperature can be explained from an increase in the energy gap between the $\mathrm{O}-\mathrm{D}$ stretch and the HDO bending 


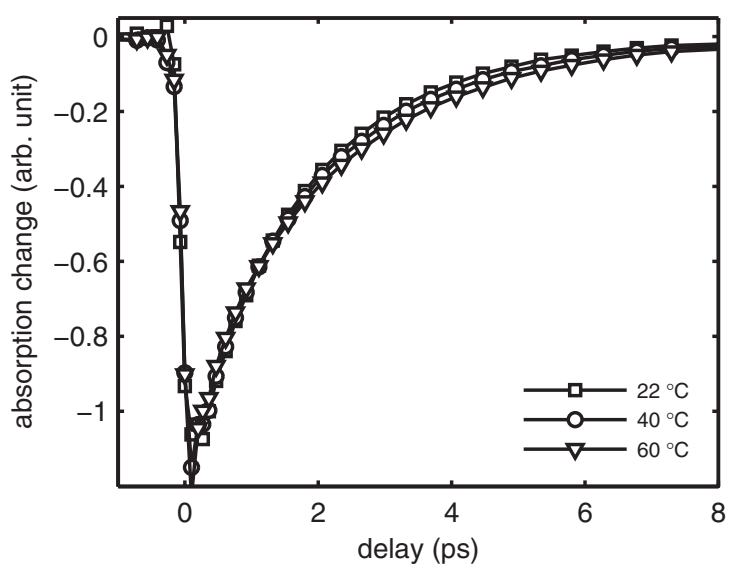

FIG. 2. Isotropic signal at the center of the O-D absorption band as a function of delay time at different temperatures for a $1.5 \mathrm{~mol} / \mathrm{kg}$ solution of TMU in HDO: $\mathrm{H}_{2} \mathrm{O}$. For all signals shown the thermalization signal has been subtracted.

mode. $^{34,35}$ The relaxation of the intermediate state is observed to become faster with increasing temperature.

\section{B. Anisotropy data}

The measured $\Delta \alpha_{\|}$and $\Delta \alpha_{\perp}$ signals contain contributions from the bleaching and induced absorption of the O-D stretch vibration, the absorption changes induced by the transient occupation of the intermediate state, and the final signal associated with the increase in temperature. The intermediate state signal represents the effect of the occupation of the intermediate state on the frequency and cross section of the previously excited O-D stretch vibration. Hence, this signal shows the same anisotropy as the bleaching and induced absorption of the excited O-D stretch vibration. The final signal associated with the increase in temperature in the focus is isotropic, meaning that the magnitude of this signal is the same for $\Delta \alpha_{\|}$and $\Delta \alpha_{\perp}$. To obtain the anisotropy of the excited $\mathrm{O}-\mathrm{D}$ vibration, the time-dependent thermalization signal has to be subtracted from $\Delta \alpha_{\|}$and $\Delta \alpha_{\perp}$. The time dependence of this signal is obtained from the modeling of the isotropic data.

In Fig. 5 the anisotropy at $2500 \mathrm{~cm}^{-1}$ is shown as a function of delay for three different concentrations of TMU

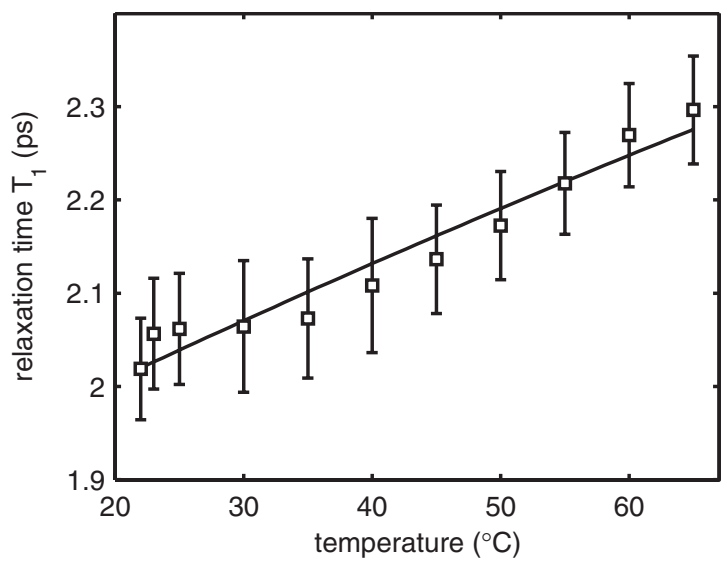

FIG. 3. Vibrational relaxation time constant $T_{1}$ as a function of temperature for a solution of $1.5 \mathrm{~mol} / \mathrm{kg}$ TMU in $\mathrm{HDO}: \mathrm{H}_{2} \mathrm{O}$.

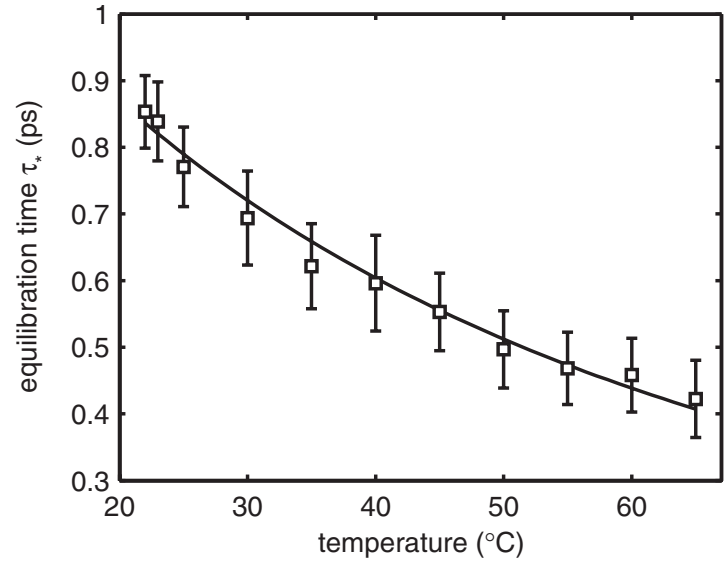

FIG. 4. Equilibration time constant $\tau_{*}$ as a function of temperature for a solution of $1.5 \mathrm{~mol} / \mathrm{kg}$ TMU in $\mathrm{HDO}: \mathrm{H}_{2} \mathrm{O}$.

at $25{ }^{\circ} \mathrm{C}$. In all cases the thermal contribution has been subtracted before calculating the anisotropy according to Eq. (2). The relaxation of the anisotropy is observed to show a fast component with a time constant of $\sim 2.5 \mathrm{ps}$ and a much slower component with a time constant $>10$ ps. The amplitude of the slow component increases with the concentration of TMU. In previous studies we have shown that at low concentrations this increase is linear, and that the amplitude of the slow component scales with the number of methyl groups contained in the solute. ${ }^{12,13}$ Hence, the slow component has been assigned to water molecules solvating the hydrophobic parts of the solute. The fast component is assigned to water molecules outside the hydration shell of TMU. Indeed, for pure HDO: $\mathrm{H}_{2} \mathrm{O}$ (no solute added) the orientational relaxation has been observed to show a time constant $\tau_{\mathrm{or}, w}$ of $\sim 2.5$ ps. $^{26-28}$

In Fig. 6 the anisotropy decay at the center of the O-D absorption band is shown for a solution of $1.5 \mathrm{~m}$ TMU at three different temperatures. We observe that increasing the temperature has a dramatic effect on the orientational relaxation. At a temperature of $40{ }^{\circ} \mathrm{C}$ the anisotropy decay shows a much faster overall decay than at $25^{\circ} \mathrm{C}$. At $65^{\circ} \mathrm{C}$ the slow component appears to have disappeared completely. For TBA we observe the same effects (not shown). The orientational dynamics of water molecules solvating the hydropho-

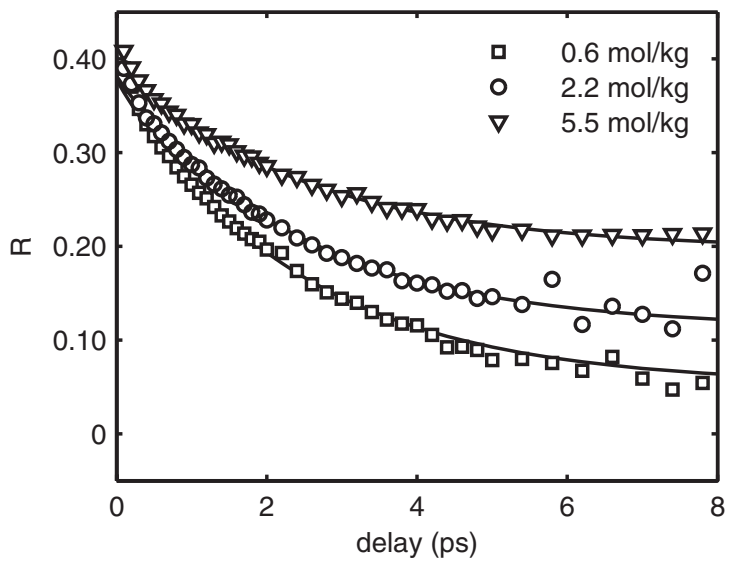

FIG. 5. Anisotropy as a function of delay time at $25^{\circ} \mathrm{C}$ for three different concentrations of TMU in HDO: $\mathrm{H}_{2} \mathrm{O}$. 


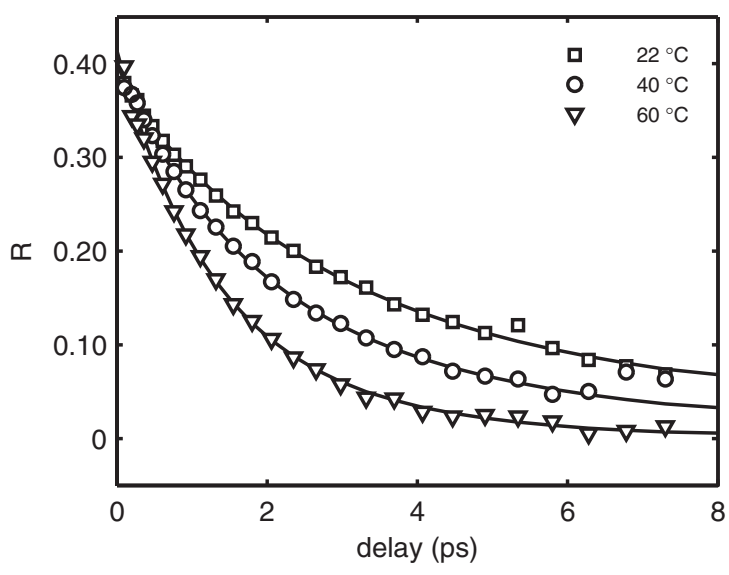

FIG. 6. Anisotropy as a function of delay for a solution of $1.5 \mathrm{~mol} / \mathrm{kg}$ TMU in HDO: $\mathrm{H}_{2} \mathrm{O}$ at three different temperatures. The solid curves are biexponential fits.

bic moieties of TMU and TBA thus appears to be strongly temperature dependent. The reorientation of the nonsolvating (bulk) water molecules will also be temperature dependent, and this temperature dependence is required to determine the value of the reorientation time constant of the solvating water molecules at different temperatures. The temperature dependence of the nonsolvating water molecules is obtained by measuring the anisotropy dynamics of pure $\mathrm{HDO}: \mathrm{H}_{2} \mathrm{O}$ (no solute added). ${ }^{36}$ The results are shown in Fig. 7.

The time constants from the analysis of the anisotropy decays of pure HDO: $\mathrm{H}_{2} \mathrm{O}$ are used in a fit of the anisotropy decays measured at different temperatures for a solution of $1.5 \mathrm{~m}$ TMU and a solution of $1.4 \mathrm{~m}$ TBA. In this fit we assume that the fraction of water molecules involved in the hydrophobic hydration does not change with temperature, i.e., we keep the amplitude of the slow component the same at all temperatures. The fractions of solvating water for the TMU and TBA solutions are found to be 0.34 and 0.37 , respectively. The resulting reorientation time constant of the solvation shells is shown in Fig. 7. It is seen that for both TBA and TMU solutions the increase in temperature leads to a dramatic decrease in $\tau_{\text {or, } s}$ from a value $>10 \mathrm{ps}$ at $25^{\circ} \mathrm{C}$ to $\sim 2$ ps at $65^{\circ} \mathrm{C}$. The reorientation of the solvating water

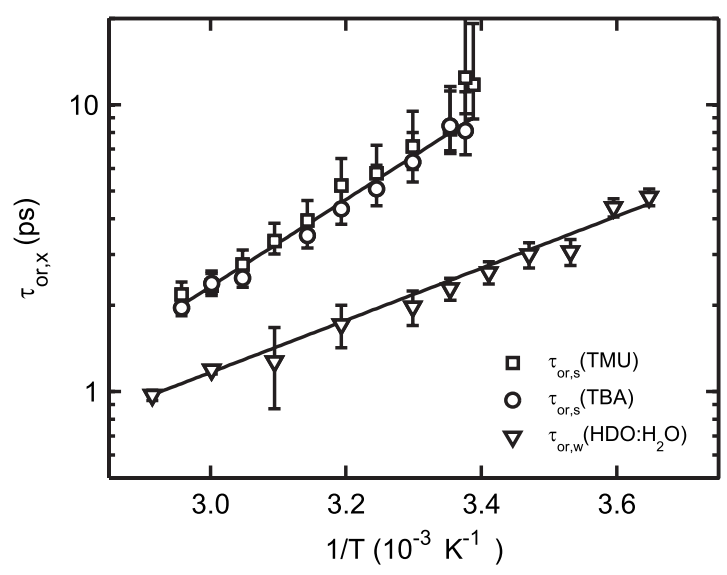

FIG. 7. Reorientation time constant $\tau_{\mathrm{or}, w}$ of pure HDO: $\mathrm{H}_{2} \mathrm{O}$ and reorientation time constants $\tau_{\text {or,s }}$ of solutions of $1.5 \mathrm{~mol} / \mathrm{kg}$ TMU in $\mathrm{HDO}: \mathrm{H}_{2} \mathrm{O}$ and $1.4 \mathrm{~mol} / \mathrm{kg}$ TBA in HDO: $\mathrm{H}_{2} \mathrm{O}$ plotted as a function of $1 / T$. The solid curves are fits to Eq. (3) describing an Arrhenius-type activated process. molecules is thus accelerated by a factor of $\sim 5$ over a temperature interval of only $40{ }^{\circ} \mathrm{C}$. The results for the TMU and TBA solutions are in excellent agreement with each other, confirming that the reorientation time constants are associated with the water molecules that interact with the hydrophobic moieties.

Assuming that the reorientation rate would be an Arrhenius-type activated process, the temperature dependence of the reorientation would be given by

$$
\tau_{\mathrm{or}, x}=A_{x} e^{-E_{\mathrm{act}, x} / k T}, \quad x=w, s,
$$

with $A_{x}$ as a pre-exponential factor, $E_{\text {act }, x}$ as the activation energy, and $k$ as Boltzmann's constant. In Fig. 7 the reorientation time constants $\tau_{\mathrm{or}, w}$ and $\tau_{\mathrm{or}, s}$ are plotted on a logarithmic scale as a function of $1 / T$. The activation energies $E_{\text {act, } w}$ and $E_{\text {act }, s}$ are given by the slopes of the resulting lines. We find $E_{\mathrm{act}, w}=17 \pm 2 \mathrm{~kJ} / \mathrm{mol}$ and $E_{\mathrm{act}, s}=29 \pm 3 \mathrm{~kJ} / \mathrm{mol}$. These activation energies agree very well with previous results of NMR. $^{6-8}$

\section{Discussion}

The temperature dependence of the orientational dynamics of water around hydrophobic groups can be explained from the effect of a hydrophobic solute on the rate and mechanism by which water molecules reorient in liquid water. ${ }^{12}$ Molecular dynamics simulations showed that the reorientation of water molecules in bulk liquid water involves the transient formation of fivefold coordinated water molecules. ${ }^{37}$ Recently, Laage and Hynes ${ }^{38,39}$ proposed a detailed mechanism for water reorientation in which one of the donated hydrogen bonds evolves into a bifurcated hydrogen bond with an additional water molecule. The switching to the new hydrogen-bonded partner leads to reorientation. It has been proposed that the hydrophobic solute hinders the formation of bifurcated hydrogen bonds, thus slowing down the reorientation. ${ }^{12}$ This effect includes two contributions. In the first place, the solute will sterically hinder the approach of a new hydrogen-bonded partner. The solute thus excludes part of the volume for forming the bifurcated transition state for reorientation. Second, the presence of the hydrophobic solute will change the hydrogen-bonded structure and structural dynamics of a number of surrounding water molecules. The hydrogen-bond network of water folds itself around the methyl groups of the solute, thereby forming a number of hydrogen bonds that are tangential to these methyl groups. The hydrogen-bond dynamics of this network can be much slower than those of bulk liquid water, thereby also making the reorientation slower.

At low concentrations, the excluded volume effect leads at most to an increase in the reorientation time by a factor of 2 (half of the volume being excluded) ${ }^{40}$ which is clearly much lower than is experimentally observed (see Fig. 5). In addition, as the molar volumes of water and TMU/TBA are not strongly temperature dependent, the excluded volume effect is not expected to depend on temperature. Hence, based on the excluded volume effect, the reorientation time of the hydration shell would be expected to show the same temperature dependence as the bulk liquid. The ratio of the re- 
orientation time constants of the solvating water decreases from a value $\sim 5$ at $25^{\circ} \mathrm{C}$ to $\sim 2$ at $65^{\circ} \mathrm{C}$. Hence, the reorientation of the hydration shell has a much stronger temperature dependence than the reorientation in bulk liquid water. The strong temperature dependence of the hydration shell reorientation is also illustrated by the high value of the activation energy $(29 \pm 3 \mathrm{~kJ} / \mathrm{mol})$, which is two times higher than the activation energy for reorientation in bulk liquid water $(17 \pm 2 \mathrm{~kJ} / \mathrm{mol})$.

The difference in temperature dependence of the hydration shell reorientation and the bulk reorientation shows that the excluded volume effect forms only a minor contribution to the slowing down of the reorientation at room temperature. Therefore, at low temperatures $\left(<50{ }^{\circ} \mathrm{C}\right)$ the observed slow hydration shell reorientation appears to be largely due to a slowing down of the hydrogen-bond dynamics of the solvating water molecules.

The activation energy for the hydration shell reorientation determined with femtosecond midinfrared spectroscopy is very similar to the values found with NMR, ${ }^{6-8}$ which implies that both techniques arrive at the same scaling of the reorientation rate with temperature. However, an important difference between the results of the two techniques is formed by the absolute values of the reorientation time constants, NMR arrives at much shorter reorientation times than femtosecond midinfrared spectroscopy. Here it should be noted that NMR only measures the average reorientation time constant of the solution, including the bulk and the hydration shell contributions. Hence, to arrive at a reorientation time constant for the water molecules hydration shell, a certain value for the solvation number has to be assumed. These numbers are obtained from molecular dynamics simulations and amount to 25 and 32 water molecules for TBA and TMU, respectively. ${ }^{9,11}$ A strong advantage of femtosecond midinfrared spectroscopy is that the bulk and the hydration shell contributions to the reorientation can be distinguished. Hence, the number of water molecules, or more precisely the number of water hydroxyl groups, that show a slowed down reorientation is directly measured. This number turns out to be significantly lower than the number of solvation shell molecules assumed in most NMR studies. For instance, for TMU only $\sim 16$ water hydroxyl groups were found to show a slow reorientation, ${ }^{12}$ which is a small number compared to the 32 water molecules (64 hydroxyl groups) of the solvation shell. ${ }^{11}$ This difference indicates that only part of the water hydroxyl groups in the solvation shell is being affected in its reorientation dynamics. In Ref. 11 it was suggested that the slow reorientation could also be due to $n>16$ hydroxyl groups showing a partial fast and a partial slow reorientation. In that case the reorientation of the hydration shell would have been strongly anisotropic, showing a fast reorientation over a limited cone angle. However, an NMR study of the spin relaxation rate of $\mathrm{D}$ and ${ }^{17} \mathrm{O}$ of $\mathrm{D}_{2} \mathrm{O}$ molecules in the hydration shell of hydrophobic molecules showed that the reorientation in the hydration shell is in fact quite isotropic (as in the bulk liquid). ${ }^{8}$ Therefore, we conclude that a hydrophobic molecular solute strongly affects the orientational dynamics of a relatively small hydrogen-bonded network of water hydroxyl groups.
The strong increase in the orientational mobility of the hydrophobic hydration shell with temperature is in line with the thermodynamics of aqueous solutions of hydrophobic molecules. With increasing temperature the excess entropy and enthalpy of the solvation increase, ${ }^{15-19}$ which suggests that the water surrounding the hydrophobic solutes becomes much less structured. Together with the present findings, it follows that the hydrogen-bond network around the methyl groups becomes increasingly dynamic at elevated temperatures, thus leading to a strong increase of the frequency of reorientation events. As a result, the difference in dynamics with the rest of the liquid strongly decreases. We observe that at $65^{\circ} \mathrm{C}$, the reorientation of the hydrophobic hydration shell and the bulk only differ by a factor of 2 . Hence, it could well be that at elevated temperatures the slowing down of the reorientation is being dominated by the excluded volume effect, meaning that the structural dynamics of the hydrogenbond network surrounding the hydrophobic groups has become similar to those of bulk liquid water.

\section{CONCLUSIONS}

We have studied the temperature dependence of the influence of TMU and TBA on the orientational dynamics of liquid water using polarization-resolved femtosecond midinfrared spectroscopy. We observe that at low temperatures ( $<30^{\circ} \mathrm{C}$ ), the water molecules solvating the hydrophobic moieties (methyl groups) of TMU and TBA show a reorientation time $>10 \mathrm{ps}$, which is approximately five times slower than the molecular reorientation in bulk liquid water. With increasing temperature, the molecular reorientation of the solvating water molecules is strongly accelerated, reaching a value of $\sim 2$ ps at $65^{\circ} \mathrm{C}$.

The reorientation of the hydrophobic hydration shell shows a much stronger temperature dependence than the reorientation of bulk liquid water. The activation energy of the hydrophobic hydration shell reorientation is $29 \pm 3 \mathrm{~kJ} / \mathrm{mol}$, which is two times higher than the activation energy for reorientation in bulk liquid water $(17 \pm 2 \mathrm{~kJ} / \mathrm{mol})$. The different temperature dependencies of the hydration shell reorientation and bulk water reorientation shows that that the slowing down of the reorientation at low temperatures is not due to an excluded volume effect. Instead, the slow orientational dynamics at low temperatures $\left(<50^{\circ}\right)$ appear to result from a slowing down of the structural dynamics of the hydrogen-bond network of the water molecules that surround the hydrophobic moieties. At an elevated temperature of $65{ }^{\circ} \mathrm{C}$, the reorientation of the hydrophobic hydration shell and the bulk only differ by a factor of 2 , which shows that the hydrogen-bond network of the water molecules surrounding the methyl groups of TMU and TBA becomes much more labile at elevated temperatures. This finding is in line with the temperature dependence of the excess entropy and enthalpy change in hydrophobic hydration that both strongly increase with temperature.

\section{ACKNOWLEDGMENTS}

This work was part of the research program of the "Stichting voor Fundamenteel Onderzoek der Materie 
(FOM)," which is financially supported by the "Nederlandse organisatie voor Wetenschappelijk Onderzoek (NWO)." We also acknowledge financial support from the Villum Kann Rasmussen Foundation. We would like to thank Mischa Bonn, Damien Laage, and Casey Hynes for useful discussions.

${ }^{1}$ H. S. Frank and M. W. Evans, J. Chem. Phys. 13, 507 (1945).

${ }^{2}$ A. K. Soper and J. L. Finney, Phys. Rev. Lett. 71, 4346 (1993).

${ }^{3}$ J. Turner and A. K. Soper, J. Chem. Phys. 101, 6116 (1994).

${ }^{4}$ S. Dixit, J. Crain, W. C. K. Poon, J. L. Finney, and A. K. Soper, Nature (London) 416, 829 (2002).

${ }^{5}$ P. Buchanan, N. Aldiwan, A. K. Soper, J. L. Creek, and C. A. Koh, Chem. Phys. Lett. 415, 89 (2005).

${ }^{6}$ M. Nakahara, C. Wakai, Y. Yoshimoto, and N. Matubayashi, J. Phys. Chem. 100, 1345 (1996).

${ }^{7}$ Y. Ishihara, S. Okouchi, and H. Uedaira, J. Chem. Soc., Faraday Trans. 93, 3337 (1997).

${ }^{8}$ K. Fumino, K. Yukiyasu, A. Shimizu, and Y. Taniguchi, J. Mol. Liq. 75, 1 (1998).

${ }^{9}$ K. Yoshida, K. Ibuki, and M. Ueno, J. Chem. Phys. 108, 1360 (1998).

${ }^{10}$ A. Shimizu, K. Fumino, K. Yukiyasu, and Y. Taniguchi, J. Mol. Liq. 85, 269 (2000).

${ }^{11}$ J. Qvist and B. Halle, J. Am. Chem. Soc. 130, 10345 (2008).

${ }^{12}$ Y. L. A. Rezus and H. J. Bakker, Phys. Rev. Lett. 99, 148301 (2007).

${ }^{13}$ Y. L. A. Rezus and H. J. Bakker, J. Phys. Chem. A 112, 2355 (2008).

${ }^{14}$ D. Chandler, Nature (London) 437, 640 (2005).

${ }^{15}$ N. Muller, Acc. Chem. Res. 23, 23 (1990).

${ }^{16}$ B. Lee and G. Graziano, J. Am. Chem. Soc. 118, 5163 (1996).

${ }^{17}$ S. Garde, G. Hummer, A. E. Garcia, M. E. Paulaitis, and L. R. Pratt, Phys. Rev. Lett. 77, 4966 (1996).

${ }^{18}$ D. M. Huang and D. Chandler, Proc. Natl. Acad. Sci. U.S.A. 97, 8324 (2000).

${ }^{19}$ N. T. Southall, K. A. Dill, and A. D. J. Haymet, J. Phys. Chem. B 106,
521 (2002).

${ }^{20} \mathrm{~S}$. Woutersen and H. J. Bakker, Nature (London) 402, 507 (1999).

${ }^{21}$ G. Gallot, S. Bratos, S. Pommeret, N. Lascoux, J.-C. Leicknam, M. Kozinski, W. Amir, and G. M. Gale, J. Chem. Phys. 117, 11301 (2002).

${ }^{22}$ A. W. Omta, M. F. Kropman, S. Woutersen, and H. J. Bakker, Science 301, 347 (2003).

${ }^{23}$ T. Steinel, J. B. Asbury, J. Zheng, and M. Fayer, J. Phys. Chem. A 108, 10957 (2004).

${ }^{24}$ M. L. Cowan, B. D. Bruner, N. Huse, J. R. Dwyer, B. Chugh, E. T. J. Nibbering, T. Elsaesser, and R. J. D. Miller, Nature (London) 434, 199 (2005).

${ }^{25}$ C. J. Fecko, J. J. Loparo, S. T. Roberts, and A. Tokmakoff, J. Chem. Phys. 122, 054506 (2005).

${ }^{26}$ Y. L. A. Rezus and H. J. Bakker, J. Chem. Phys. 123, 114502 (2005).

${ }^{27}$ Y. L. A. Rezus and H. J. Bakker, J. Chem. Phys. 125, 144512 (2006).

${ }^{28}$ I. R. Piletic, D. E. Moilanen, D. B. Spry, N. E. Levinger, and M. D. Fayer, J. Phys. Chem. A 110, 4985 (2006).

${ }^{29}$ S. Park and M. D. Fayer, Proc. Natl. Acad. Sci. U.S.A. 104, 16731 (2007).

${ }^{30}$ D. E. Moilanen, E. E. Fenn, Y.-S. Lin, J. L. Skinner, B. Bagchi, and M. D. Fayer, Proc. Natl. Acad. Sci. U.S.A. 105, 5295 (2008).

${ }^{31}$ R. L. A. Timmer and H. J. Bakker, J. Chem. Phys. 126, 154507 (2007).

${ }^{32}$ A. J. Lock and H. J. Bakker, J. Chem. Phys. 117, 1708 (2002).

${ }^{33}$ S. Woutersen, U. Emmerichs, H.-K. Nienhuys, and H. J. Bakker, Phys. Rev. Lett. 81, 1106 (1998).

${ }^{34}$ R. Rey and J. T. Hynes, J. Chem. Phys. 104, 2356 (1996).

${ }^{35}$ C. P. Lawrence and J. L. Skinner, J. Chem. Phys. 119, 1623 (2003).

${ }^{36}$ K. J. Tielrooij, C. Petersen, Y. L. A. Rezus, and H. J. Bakker, Chem. Phys. Lett. 471, 71 (2009).

${ }^{37}$ F. Sciortino, A. Geiger, and H. E. Stanley, Nature (London) 354, 218 (1991).

${ }^{38}$ D. Laage and J. T. Hynes, Science 311, 832 (2006).

${ }^{39}$ D. Laage and J. T. Hynes, Chem. Phys. Lett. 433, 80 (2006).

${ }^{40}$ D. Laage, G. Stirneman, and J. T. Hynes, J. Phys. Chem. B 113, 2428 (2009). 